\title{
Identity construction through gendered terms of addresses in Korean
}

\author{
Sunwoo Jeong \& Seong-hyun $\mathrm{Yu}^{*}$
}

\begin{abstract}
How should a speaker call a hearer? In this paper, we present an experimental study which probes the social and interactional meaning of Korean gendered terms of addresses (GTAs: unnie, oppa, noona, hyung). GTAs prescriptively index genders of both interlocutors, but are beginning to be used in 'gendermismatch' patterns. Based on the experimental results, we argue that both the prescription conforming and the 'mismatching' uses of GTAs are each associated with unique, complex webs of meanings which track the shifting gender ideologies in Korea. In particular, mismatching uses of GTAs are shown to often function as speakers' strategy to break away from established gender norms, including traditional gender roles and the sexualization of female-male relations.
\end{abstract}

Keywords. terms of addresses; language and gender; Korean; social meaning; language ideologies

1. Introduction. How do we call each other, and what does the answer reveal about our identities and the relationships we form in a society? Other than names, we also often use 'terms of addresses' to call or refer to someone else. These include terms such as dude, sis, honey, your honor, madame $\mathrm{X}, \mathrm{Mr} \mathrm{Y}$, etc. Terms of addresses are particularly interesting from a sociolinguistic perspective, because they reveal not just speaker-hearer relationships, but also the evolution of the broader social structures that prescribe the roles we take on and relationships we enter into in a given community.

The specific terms of address that we are interested in are familial, gendered terms of addresses in Korean, henceforth GTAs. Here, we focus on 4 such GTAs, unnie, oppa, noona, and hyung. GTAs are ubiquitous in everyday interaction, as well as in mainstream Korean media. They are also one of the first expressions that international fans of K-dramas and K-pop familiarize themselves with, and actively take on as a part of their own language practice. The full dimensions of social meanings associated with these GTAs however, have not yet been thoroughly investigated. The aim of this paper is to clarify the evolving meanings associated with the 4 GTAs and situate them within the changing fabric of the Korean society.

1.1. ORIGIN AND USE. Etymologically, the 4 GTAs in question functioned as kinship terms and were only used to address one's older siblings (i.e., older brothers and sisters). This basic function is maintained to the present day, but now, GTAs also have a much wider usage pattern. A first pass empirical generalization is that GTAs can be used to address or refer to basically any (slightly) older person that one feels close to, like an older friend or an older romantic partner. The age gap needs to be below a certain threshold however, as other more deferential terms should be used for hearers who are much older than the speaker. Native speaker intuitions suggest that the critical threshold is around 10 years of age gap (with more leeway granted if the speaker and the hearer are very close).

* This work was supported by the New Faculty Startup Fund (Sunwoo Jeong) and the Student Directed Education Fund (Seonghyun Yu) from Seoul National University. We thank Robert Podesva, Penny Eckert, Mi-suk Lee, and the audience at the LSA 2021 annual meeting for insightful comments and questions. We also thank the experimental participants for their time and insights. Any remaining errors are our own. Authors: Sunwoo Jeong, Seoul National University (sunwooj@snu.ac.kr) \& Seonghyun Yu, Seoul National University (ehdrmfd11021@ naver.com). 
The 4 GTAs can be used in isolation as in (1), or they can be used combined with the first name of the person as in (2) to address or call attention to someone. Outside their vocative uses, they also have referential uses as in (3), though in this paper, we will focus more on their uses as terms of addresses. In examples (1)-(3), loose translations onto cultural analogues (in English) have been provided in the last lines. ${ }^{1}$

\section{(1) Unnie!}

Sister!

₹ 'FIRSTNAME!' (addressing a close, slightly older friend)

(2) Byul unnie!

Byul sister!

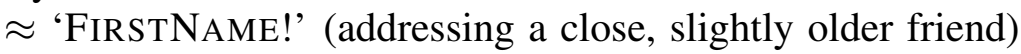

(3) na ecey Byul unnie-lul manna-ss-e.

I yesterday Byul sister-ACC meet-PST-DECL.

$\approx$ 'I met Byul (a close, slightly older friend) yesterday.'

1.2. GTAS AND GENDER. One particularly interesting aspect of the Korean GTA system is that it is quite intricately subdivided depending on both speaker and hearer genders, and that these gender-term correspondences appear to function as strong prescriptive norms.

Specifically, when the speaker and the hearer are both female, one is expected to use unnie. When the speaker is female, and the hearer male, one is expected to use oppa. When the speaker is male and the hearer female, one is expected to use noona. Finally, when the speaker and the hearer are both male, one is expected to use hyung. This state of affairs is summarized in Table 1.

\begin{tabular}{l|lc}
\hline Speaker & Female $(\mathrm{F})$ & Male $(\mathrm{M})$ \\
\hline Hearer & unnie $(\mathrm{FF})$ & noona $(\mathrm{MF})$ \\
Male(M) $(\mathrm{M})$ & oppa $(\mathrm{FM})$ & hyung $(\mathrm{MM})$ \\
\hline
\end{tabular}

Table 1. GTAs and interlocutor gender

The reason why GTAs are so ubiquitous in Korea may be due to the combination of two key factors: First, culturally, some form of marking that clarifies the seniority of the hearer is more or less required whenever calling on someone older in age, no matter how small the age gap may be, and how close and informal the relationship may be. Second, when it comes to calling on someone older with whom one has a close, familiar relationship, the space of available alternatives appears to be pretty narrow. If one doesn't opt for the 4 GTAs, then one is stuck with other honorific titles like ssi (which is analogous to Mr or Ms in English), nim, or sunbae, which sound quite formal and a bit distant. At the same time, as noted earlier, opting to call someone older just by their first name is still considered by many Koreans to be quite rude and inappropriate. Consequently, GTAs end up being more or less the only option which

\footnotetext{
${ }^{1}$ We follow the Yale Romanization conventions in the examples, with the exception of proper names $(B y u l)$ and the 4 GTAs themselves (unnie, oppa, noona, hyung). Regarding the proper names, we adopt the romanization conventions standardly used by the people with the said names; regarding the GTAs, we adopt the romanization forms most widely used by both Korean and non-Korean speakers in interactions conducted in English on web forums, etc.
} 
can do double duty: (i) clarify the seniority of the hearer and (ii) signal the relatively close and informal nature of the relationship.

It is worth mentioning here that the norms for addressing younger siblings, friend, or partners are subject to much less constraints: when addressing a close hearer who is younger, it is fine to just call them by their first names, as in English. In sum, the intricate prescriptive system outlined in Table 1 appears to target just the older hearers that one feels close to.

1.3. The outline. What core meanings, including denotational, referential, interactional, and social, do the 4 GTAs contribute? At first glance, one might think the answer to this question is pretty straightforward. Summarizing the initial intuitions we've established so far, one may posit that all 4 GTAs share the key function of conveying two kinds of relational meanings, namely, (i) that the social distance between the speaker and the hearer is below a certain threshold $\theta$, i.e., the speaker and the hearer are close, and (ii) that the hearer is older than the speaker by a certain margin (not more than 10 years). One may then posit that each GTA encodes 4 different interlocutor gender possibilities assuming binary gender ${ }^{2}$ and a dyadic interaction. In sum, one may provide working semantics like the one outlined in Table 2 for the 4 GTAs.

$$
\begin{gathered}
\text { unnie }=\left[\begin{array}{ccc}
\text { dist(spkr, hrer) } & < & \theta \\
\text { age(spkr) } & < & \text { age(hrer }) \\
\text { gender(spkr) } & = & \mathrm{F} \\
\text { gender(hrer) } & = & \mathrm{F}
\end{array}\right] \quad \text { oppa }=\left[\begin{array}{ccc}
\text { dist(spkr, hrer) } & < & \theta \\
\operatorname{age}(\mathrm{spkr}) & < & \text { age(hrer }) \\
\text { gender(spkr) } & = & \mathrm{F} \\
\text { gender(hrer) } & = & \mathrm{M}
\end{array}\right] \\
\text { noona }=\left[\begin{array}{ccc}
\text { dist(spkr, hrer) } & < & \theta \\
\text { age(spkr) } & < & \text { age(hrer }) \\
\text { gender(spkr) } & = & \mathrm{M} \\
\text { gender(hrer) } & = & \mathrm{F}
\end{array}\right] \quad \text { hyung }=\left[\begin{array}{ccc}
\text { dist(spkr, hrer) } & < & \theta \\
\operatorname{age}(\mathrm{spkr}) & < & \text { age(hrer) } \\
\text { gender(spkr) } & = & \mathrm{M} \\
\text { gender(hrer) } & = & \mathrm{M}
\end{array}\right]
\end{gathered}
$$

Table 2. A possible semantics for the 4 GTAs

In the remainder of the paper, we demonstrate that this simplistic view glosses over the complex web of meanings associated with the 4 GTAs. Relying on native speaker intuitions, some corpus examples, and a pilot experiment, we establish the following new observations: (i) A given GTA is associated with a wider range of additional meanings that are unique to each. (ii) These meanings reflect the dominant gender ideologies of Korea, including traditional gender roles and gender relations. (iii) People sometimes also use GTAs in non-prescriptive ways, resulting in both age mismatching and gender mismatching uses. (iv) Such mismatching uses can come to signal additional salient social meanings and can provide speakers with ways of breaking away from established gender norms.

2. Asymmetries in GTA meaning. We begin by making a case that there exists certain semantic asymmetries between the 4 GTAs which go beyond mere interlocutor gender differences. First, the potential for signaling deference appears to be asymmetrically encoded: GTAs like hyung and noona, which prescriptively index male speakers, can combine with an additional honorific marker nim, as in hyung-nim or noon-im, thereby enabling the speaker to con-

\footnotetext{
${ }^{2}$ This, as one might expect, is not reflective of the reality, and erases non-binary people. See also sec. 6 .
} 
vey both affinity and deference. By contrast, analogous options do not appear to exist for unnie and орра, which prescriptively index female-speakers (\#oppa-nim and \#unnie-nim are infelicitous; orabeu-nim may be construed as a term which can fill in the gap of \#oppa-nim, but is construed to be archaic, and is thus no longer used by younger speakers). This appears to reflect the dominant preconception that the dynamics of close relationships with older people is realized a bit differently depending on the gender: for male speakers, conveying a particular kind of deferential attitude is perhaps as important as (or more important than) conveying affinity, even in close relationships.

The social capital granted by the deferential variants of the GTAs, hyung-nim or noon-im, is characterized by culture-specific flavors formulated around masculine loyalty and affection. Notably, the director Bong Joon-Ho thanked Quentin Tarantino in his 2020 Oscar (best director) acceptance speech by addressing him as Quentin-hyungnim, as in (4). Presumably, Bong was aiming to transpose a uniquely Korean kind of brotherhood, which is simultaneously extremely affectionate but also deferential.

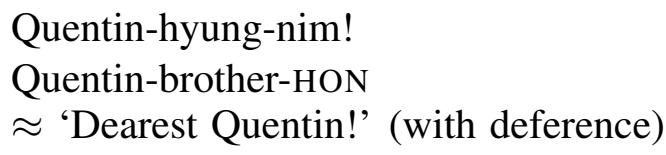

From Bong Joon-Ho's 2020 Oscar acceptance speech (Best Director)

Not just the potential for masculine deference, but also the potential for derogation appears to be asymmetrically encoded depending on the GTA. Perhaps less so now, but at some point in time, the GTA unnie (a female to female address term) was used as a lingo to refer to sexual workers in a derogatory way (Kim 2008). The semantic derogation of initially neutral female terms of addresses is a widely documented phenomenon (see e.g., Eckert \& McConnell-Ginet 2013; Kim 2008). The case of unnie suggests that Korean is not an exception, and demonstrates that the term unnie can be associated with an additional layer of meaning, namely, that of derogation, which is absent in say, noona or hyung.

In a somewhat different vein, the term oppa has also obtained a kind of derogatory layer of meaning absent in the other GTAs (see also sec. 6). This layer of meaning appears to have originated in the K-pop fandom, likely made salient via the accumulation over time of what we'll call 'your oppar' memes: (5) provides an example from Twitter.

(5) "She's hotter than the oppars out there but please don't call her your oppar, it's a downgrade."

A tweet by@byuliesus on April 30th, 2017; accessed on December 12th, 2020

Essentially, 'your oppar' memes provide a way of poking fun at hyper-enthusiastic fan girls, as well as their targets of adoration, i.e., male K-pop stars. The derogatory use of 'oppa(r)' appears to have been frequently invoked in the female star K-pop fandoms, which are generally more marginalized than the male-star ones. Nowadays however, it is more generally invoked in fan wars including male-to-male ones (e.g., "your oppar could never"). The addition of ' $r$ ' in 'oppa' appears to be deliberate and is likely a way of deriding uninitiated, young international fans. (In Korean, rhotic $\mathrm{r}$ is phonotactically ill-formed in the coda position, and 'oppar' is thus perceived as an infelicitous, anglicized pronunciation.) Interestingly, those who invoke the term 'oppar' or the associated memes often appear to be international (non-Korean) fans as well. In sum, oppa(r) (in particular its referential uses) appears to have obtained a 
derogatory layer of meaning, where the target of derogation is not just the referent, but also the hearer who would function as the speaker of the said GTA in other situations. The emerging meaning appears to reflect the perception that the dynamics between a younger female and an older male can be one-sided and potentially belittling towards the hearer.

3. Mismatch uses of GTAs. We have so far argued that each of the 4 GTAs may contribute unique, asymmetric meanings which likely reflect the dominant gender ideologies of the language community. We now propose that the prescriptive norms regarding speaker and hearer age and gender summarized in Table 1 and implemented in Table 2 may be violated in actual uses of the GTAs, and that such mismatching uses are associated with additional layers of emergent meaning.

3.1. Age Mismatch USES. First, contra the prescriptive norm, certain GTAs can be used to address someone younger than the speaker. This is often done to compliment the charisma of the hearer and/or express (quasi-)romantic feelings towards them. For example, various adaptations of a formula along the vein of "I'm older than you, but since you are cooler or handsomer than me, you are unnie to me, or you are oppa to me." can be easily found on the internet (6)-(7), often as comments to performance videos of a star. For instance, a female fan of a famous, charismatic Korean actress Kim Hye-Soo took this formulaic comment to the extreme, and sent a jokey fan letter shown in (8). To the amusement of the interviewer who read the fan letter, Kim Hye-Soo actually mistook the fan as a child, and responded with "Oh, my God! What a cute baby! Let's send her a present."

"I'm 4 years older than you. Can I call you oppa?"

Adapted from YouTube comments expressing similar sentiments

(7) "Since you are cooler/handsomer than me, you are unnie."

Adapted from tweets expressing similar sentiments

(8) "Unnie, I love you I'm 8 years old (serious)."

From an interview of Kim Hye-Soo

Interestingly, age-mismatching uses of GTAs appear to be restricted to female speakers (and terms indexing them, unnie and oppa). We haven't yet come across male speakers using noona or hyung in comparable age-mismatching ways, i.e., to address younger hearers while expressing an awed, deferential attitude or quasi-romantic feelings (the latter appears to be especially missing, likely because seniority and deference are often perceived to figure less in male speakers' relations to their romantic partners).

3.2. Gender mismatch USES. Second, contra the prescriptive norm, GTAs are sometimes used in 'gender mismatching' scenarios as well. Initial native speaker intuition suggests that such uses are more commonly available for GTAs which index same gender relations such as unnie (female to female) or hyung (male to male).

For instance, the term unnie appears to be sometimes used by males in a female dominated community, industry, or interaction, such as a male salesperson in a cosmetic store addressing a female customer (9).

(9) A male seller addressing a female customer:

"Unnie!" 
The term hyung as well, is sometimes used by female speakers to address a close male friend, or even a close female friend (10).

(10) A female speaker addressing an older male friend: "Hyung!"

The use of hyung by female speakers is widely acknowledged by older generations (currently in their 50s-70s) to have been a common practice among female social activists in University campuses, particularly during the era in which demonstrations were frequent. In the present era, hyung appears to be used more widely by female speakers to emphasize the chill or tough nature of the speaker, and/or the platonic, non-romantic nature of her relationship with the male hearer (see sec. 6 for more discussion).

4. Emerging questions. To recapitulate, the 4 GTAs appear to be associated with complex constellations of interactional and social meanings which go beyond signaling closeness and indexing interlocutor age and genders according to the prescription (Table 1).

Based on these emerging intuitions, we formulated the following more targeted research questions regarding the 4 GTAs: First, what are the indexical fields, in the sense of Eckert (2008), associated with the 4 GTAs? Second, what are some salient asymmetries in meanings between the 4 GTAs? Do the ones we've identified so far (sec. 3) hold water upon closer inspection? Third, what types of meanings do mismatching uses of GTAs bring about, and more fundamentally, when and why do such mismatches occur? Finally, how are GTA uses influenced by dominant gender norms and preconceptions on gender relations, and if at all possible, how can GTA uses in turn influence or counteract such conceptualizations?

To address these questions, we conducted a pilot perception experiment. In the interest of space, moving forward, we will focus primarily on 'gender mismatch' uses but not 'age mismatch' uses of the GTAs.

5. A pilot experiment. In the experiment, participants were given minimal dialogue scenarios where a speaker was calling attention to the hearer. After familiarizing themselves with each scenario, participants answered a range of questions inquiring about the perceived characteristics of the speaker, the likely relationship between the speaker and the hearer, etc.

The general design of the experiment was inspired by Kiesling (2004)'s study on dude, as well as a variety of matched-guise sociolinguistic experiments such as Campbell-Kibler (2007), among others.

5.1. Methods. Let us briefly go over in more detail the design and implementation of the experiment. The experiment (hosted online), the associated codes, and the stimuli files can be found in the link provided in sec. 7.

5.1.1. PARTICIPANTS. The participants in our experiment were 30 native speakers of Korean, recruited via a snowball sampling method. All of them self-identified as cis-female or cis-male, and most of them were in their 20s. We did not inquire about the sexual orientations of the participants - we plan to incorporate this question in a follow-up study.

5.1.2. STIMULI. In the experiment, a total of 5 terms of addresses were tested: the 4 GTAs in question (henceforth target cases), as well as one none-GTA alternative, first name $+s s i$, which, as mentioned earlier, is analogous in flavor to English $\mathrm{Mr}$ or $\mathrm{Ms}$ (henceforth baseline cases). 
These 5 terms of addresses were presented inside a speech bubble associated with one of the 4 possible minimal dialogue scenarios summarized in Figure 1. As shown in Figure 1, in each scenario, only the genders of the interlocutors and the choice of a GTA (inserted in the speech bubble) were given. ${ }^{3}$
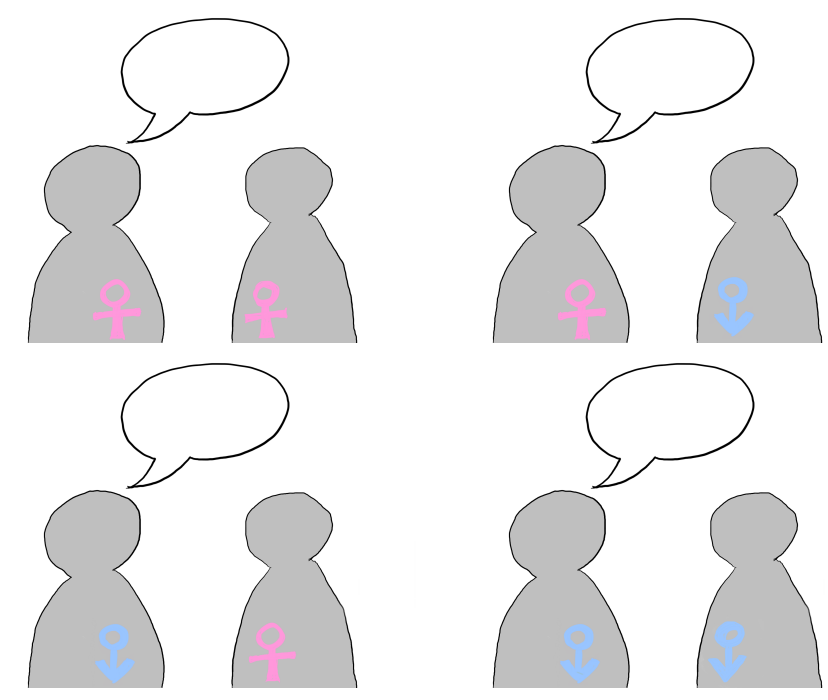

Figure 1. Stimuli template used in the experiment

Since we are interested in not only prescription-conforming, 'matching' uses of GTAs, but also mismatching uses of GTAs, the 4 gender scenarios outlined in Figure 1 were fully crossed with the 4 target GTAs, resulting in 16 types of GTA use scenarios summarized in Table 3. Likewise, the 'first name $+s s i$ ' address use can be paired with any of the 4 scenarios in Figure 1 , adding 4 more types of scenarios. Consequently, a total of 20 possible stimuli (16 target cases +4 baseline cases) were created.

As shown in Table 3, for each GTA, we could in theory have a speaker mismatch scenario (apricot orange), hearer mismatch scenario (mint green), and both speaker and hearer mismatch scenario (orchid purple). In practice, probably only a subset of these uses are common, but we decided to test all in the present experiment.

\begin{tabular}{l|cccc|cccc}
\hline \multicolumn{1}{c|}{ Spkr } & \multicolumn{4}{|c|}{ Female(F) } & \multicolumn{4}{c}{ Male(M) } \\
Hrer & \multicolumn{1}{c}{ unnie } & noona & oppa & hyung & noona & unnie & hyung & oppa \\
Female(F) & $\begin{array}{c}\text { oppa } \\
\text { hyle(M) }\end{array}$ & hyung & unnie & noona & hyung & oppa & noona & unnie \\
\hline
\end{tabular}

Table 3. Possible scenarios speaker mismatch: hearer mismatch: both mismatch:

5.1.3. ProcedURE. In each trial, participants saw a particular GTA use embedded in a dialogue scenario from Figure 1. They then answered a series of questions. In the interest of space, we will concentrate on only a subset of these questions in the paper. The first set of

\footnotetext{
${ }^{3}$ For the present experiment, we restricted ourselves to the 4 types of gender combinations shown in Figure 1. Additional possibilities would arise if we include non-binary speakers/hearers.
} 
questions we will focus on is summarized in (11). These inquired about the likely characteristics of the speaker. Specifically, participants rated how well descriptions like tultul (which roughly refers to a chill, down to earth temperament), aegyo (which roughly refers to a cute, infantile, feminine displays or characteristics; see e.g., Moon (2017) for a more detailed characterization), extroversion, politeness, femininity, etc., would fit the speaker portrayed in the dialogue. These traits were selected to be included in the experiment based on informal interviews with our friends and our own intuitions about the salient social effects of the 4 GTAs.

(11) Q1 How well do you think the following descriptions would fit the speaker?
a. Is tultul $(\approx$ chill, down-to-earth $)$
b. Has aegyo $(\approx$ cute $)$
c. Is extroverted
d. Is polite
e. Is feminine
f. Is masculine

The second set of questions we will focus on is summarized in (12). These probed the likely relationship between the speaker and the hearer. Specifically, participants rated how likely it is that the interlocutors are siblings, romantic partners, close friends, work friends, acquaintances, or strangers. Relatedly, they also answered some stance-related questions, including the one inquiring about the perceived closeness with the hearer from the point of view of the speaker (13-a).

(12) Q4 How likely is it that the interlocutors have the following relationship?
a. Siblings
b. Romantic partner
c. Close friend
d. Work friend
e. Acquaintance
f. First encounter

(13) Q5 How likely is it that the speaker has the following attitude towards the hearer?

a. Feels close to the hearer

b. Has a positive opinion about the hearer

All questions outlined so far incorporated a 7-point rating scale in terms of the possible replies.

The final set of questions we will focus on tapped into extensions of the provided scenarios onto participants' personal experiences and impressions. Specifically, as summarized in (14), participants rated how often they have personally encountered GTA uses like the one portrayed in the picture. In addition, as summarized in (15), they were invited to elaborate further on the specific scenarios in which they have encountered such uses. Relatedly, they were also invited to leave any additional comments that they think might be relevant, as in (16).

(14) Q6 How often have you personally experienced address uses like the one above?
a. Not at All
b. Sometimes
c. Often 


\section{d. frequently}

(15) Q7 In what specific situations did you experience address uses like the one above?

(16) Q8 Any other comments or thoughts?

In the experiment, each participant saw a total of 5 trials. These included 4 target trials, whereby each of the 4 GTAs appeared exactly once, and each of the 4 match/mismatch scenarios appeared exactly once as well. The pairings between the GTAs and the match/mismatch types were randomized across participants. The trials also included 1 baseline trial involving ssi, paired randomly with 1 of the 4 possible (gendered) dialogue scenarios. The ordering of trials were randomized.

5.2. RESUlTS. We now turn to the key experimental results. Regarding the answer to each type of forced-choice response questions, mixed effects ordinal regression models were fitted with participants' responses as the key dependent variable and GTA type (unnie, oppa, noona, hyung), (mis)match type, and participant gender as independent variables, with random intercepts for participants. In the interest of space however, we gloss over the details of the statistical analyses moving forward, and report only on high-level generalizations supplemented by relevant visualizations. More details on the model summaries as well as the full data set in .csv can be found in the link provided in sec. 7.

5.2.1. USAGE \& FREQUENCY. We'll begin with the results for the question outlined in (14), namely, the one involving participants' personal experience with GTA uses. In Figure 2, each panel represents the 4 types of gender match/mismatch scenarios: matching uses of GTAs, speaker mismatching uses of GTAs, hearer mismatching uses of GTAs, and both speaker and hearer mismatching uses of GTAs. The y-axes map the mean frequency of GTA encounter selected by the participants (i.e., their answers to (14)), with error bars representing standard errors. The colors and the X-axes represent the 4 GTAs: unnie, oppa, noona, and hyung.

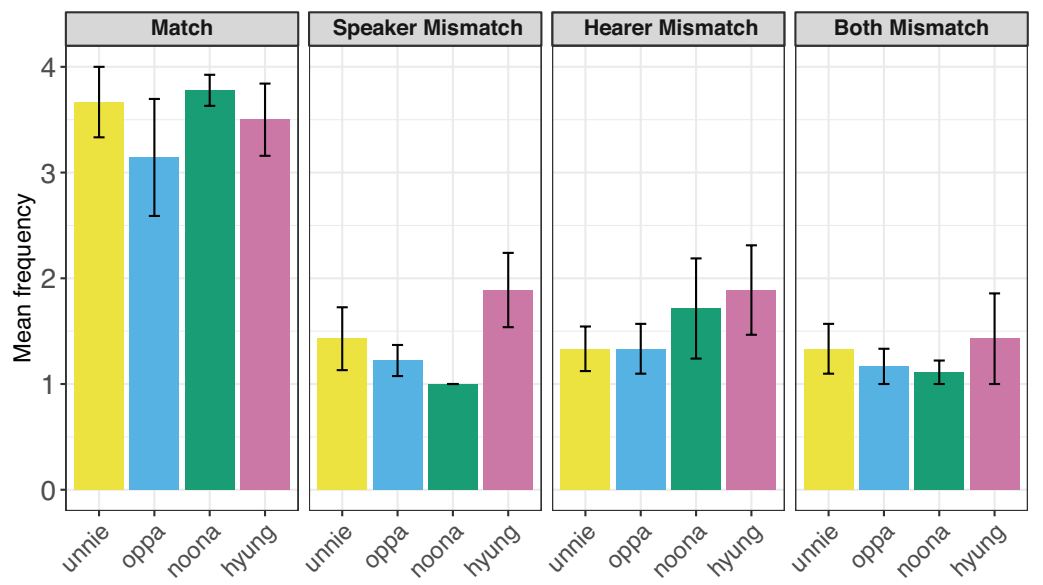

Figure 2. Frequency of personal encounters with matching and mismatching GTA uses

Legend: unnie: oppa: noona: hyung: ssi:

As can be seen from Figure 2, in the case of prescription-conforming, matching uses (the first panel), participants appear to have encountered all 4 types of GTAs quite frequently. The 
GTA oppa (the prescriptively female to male addressing term) is perhaps slightly less frequently encountered than the others, but not by much.

By contrast, in the case of mismatching uses (the three other panels), participants don't appear to have had much personal experiences with any of the 3 possible mismatch scenarios. This is a potential limitation of the study, since we are especially interested in more deeply investigating these mismatching uses. It likely reflects the fact that the current participant pool does not include enough participants who are more prone to actively use GTAs in mismatching ways. (See sec. 6 for more discussion.) Somewhat offsetting this problem however, many participants also commented in their answers to (15) or (16) that although they haven't personally used or encountered them, they are aware that such mismatching uses exist, and know of others who might come into more regular contacts with them.

Among the mismatching uses, hyung (the prescriptively male to male addressing term) appears to have been relatively more frequently encountered than the other terms, regardless of the specific mismatch type (speaker, hearer, or both). This is indicated by the consistently higher pink bars in all 3 panels on the right.

5.2.2. Shared MEANings of GTAs. Let us now turn to the results which confirm our initial intuitions about the core function shared by all 4 GTAs. Figure 3 summarizes participants' ratings on the degree of closeness between the speaker and the hearer (the first panel), the likelihood that the hearer is a (mere) acquaintance of the speaker (the second panel), and the perceived politeness of the speaker (the third panel).

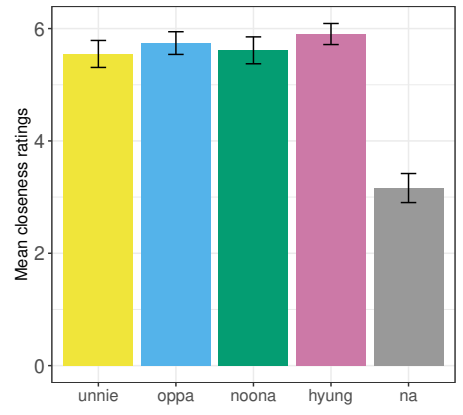

(a) Degree of closeness

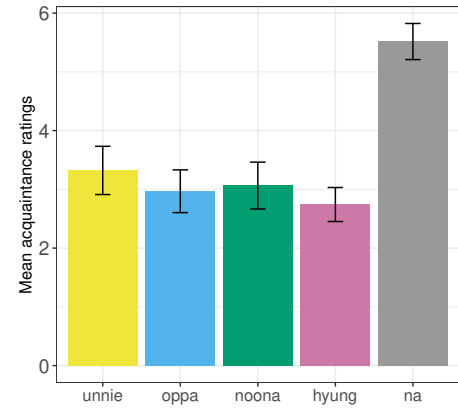

(b) Acquaintance likelihood

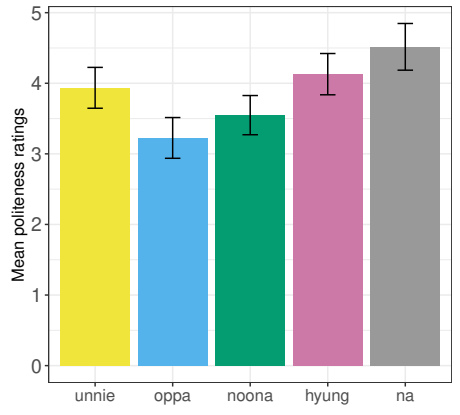

(c) Politeness

Figure 3. Commonalities across the 4 GTAs

Legend: unnie: oppa: noona: hyung: ssi:

First, as captured in the first panel, participants attributed significantly higher closeness ratings to all 4 GTAs than the baseline alternative, ssi $(\approx \mathrm{Mr}, \mathrm{Ms})$. Conversely, as captured in the second panel, ssi obtained significantly higher acquaintance ratings than the 4 GTAs. Finally, as captured in the third panel, the 4 GTAs obtained marginally lower politeness ratings than ssi, with oppa being perceived as the least polite.

The results on closeness ratings and acquaintance ratings corroborate our starting intuition (summarized in sec. 1.3) that the 4 GTAs encode a kind of close, affectionate, and informal relationship between the speaker and the hearer (in particular, closer and more informal than the relationship cued by alternatives such as ssi). The results on politeness ratings further suggest that such functions of GTAs may sometimes result in a perceived trade off in politeness. 
5.2.3. Asymmetries IN GTA MEANing. Let us now turn to the results which capture the differences and asymmetries in meanings between the 4 GTAs, starting with the relational dimension. Figure 4 summarizes participants' answer to (12). Each of the 4 panels represent the likelihood ratings for the interlocutors being in (i) an actual sibling relationship, (ii) a romantic partner relationship, (iii) a close friend relationship, and (iv) an acquaintance relationship.

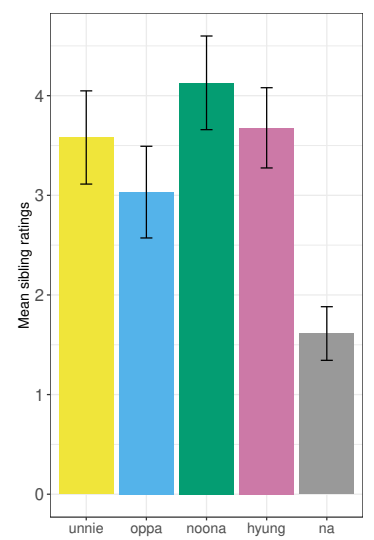

(a) Sibling

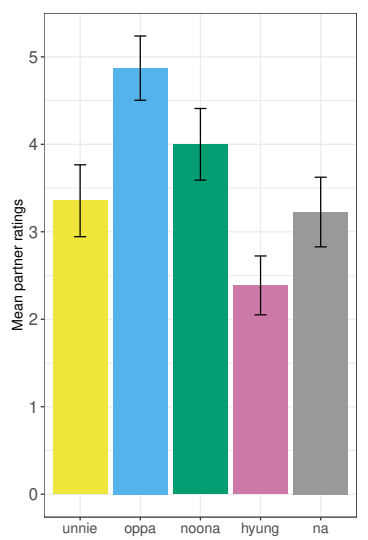

(b) Partner

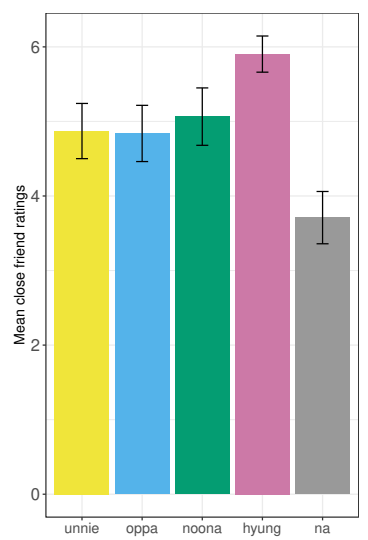

(c) Close friend

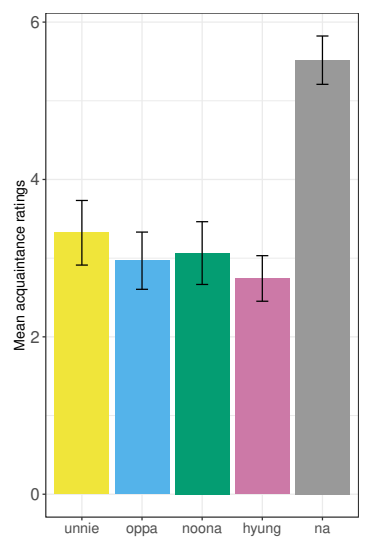

(d) Acquaintance

Figure 4. Likely relationship between the interlocutors

Legend: unnie: oppa: noona: hyung: ssi:

As can be seen from Figure 4, each of the 4 GTAs appear to be biased towards encoding different kinds of relationships, with oppa (the prescriptively female to male addressing term) being most likely to be used in romantic relationships, and hyung (the prescriptively male to male addressing term) being more likely to be used in close friend relationships. Specifically, oppa (the blue bar) obtains the highest romantic partner rating (the second panel), and hyung (the pink bar) obtains the highest close friend rating (the third panel).

As we already saw in Figure 3, all 4 GTAs are also much less likely to signal mere acquaintance relations than ssi (the fourth panel). In addition, all 4 GTAs are still likely to signal actual sibling relationships (the first panel), though to a somewhat lesser extent for oppa.

The association between a particular GTA (e.g., hyung) and a particular type of relationship appears to be general in that they hold somewhat irrespective of the actual genders of the interlocutors (and also irrespective of gender match or mismatch types). Specifically, we may break down the results for romantic partner ratings and close friend ratings further, depending on speaker and hearer genders, as shown in Figure 5. Here, rows of panels represent speaker genders, and columns, hearer genders.

In the case of romantic partner ratings (the left section), the lower left panel represents female to male interactions - and it is expected that the prescription conforming орра (the blue bar) obtains the highest romantic partner rating. However, even in the case of female to female interactions (the upper left panel), and male to male interactions (the lower right panel), i.e., even in potentially lesbian or gay relationships, oppa still obtains the highest romantic partner ratings - higher than unnie or hyung, which would have been the prescription conforming choices, respectively. Only in the case of male to female interactions (the top right panel) does the prescription conforming noona gains ascendancy over oppa. This suggests that 


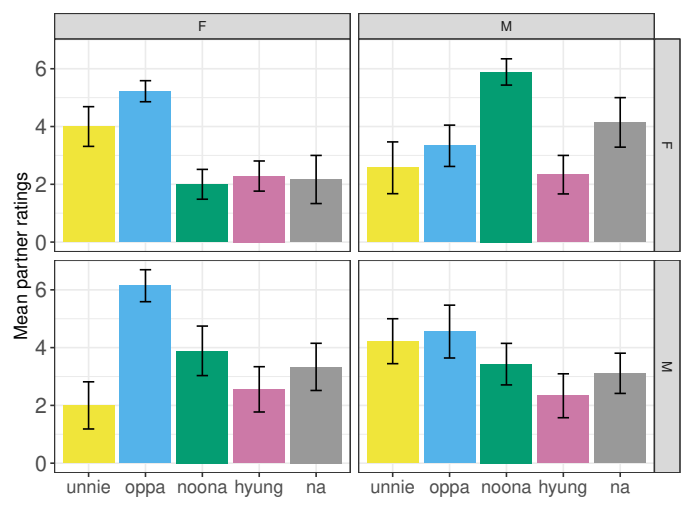

(a) Partner

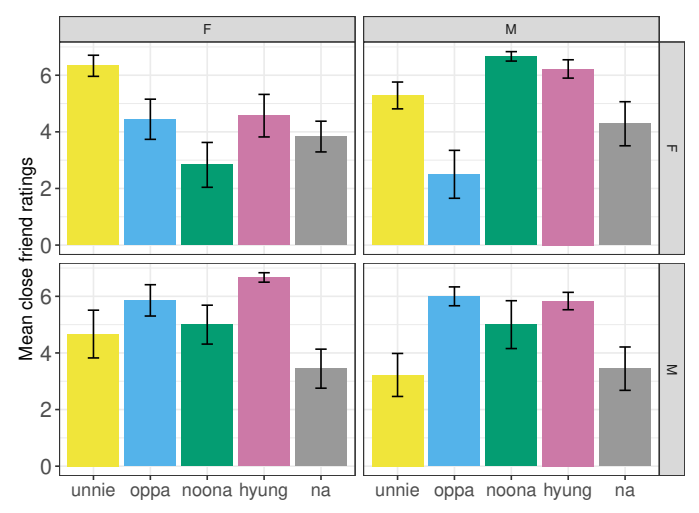

(b) Close friend

Figure 5. Likely relationships and interlocutor genders Legend: unnie: oppa: noona: hyung: ssi:

the GTA oppa may, depending on the situation, have an extended semantic effect of signaling romantic relationships in general (somewhat irrespective of speaker and hearer gender).

A parallel kind of generalization emerges for hyung in the case of close friend ratings (the right section). Here again, it is expected that the matching use of hyung (the pink bar) obtains a high close friend rating in male to male interactions (the lower right panel). Beyond that however, hyung also obtains high friend ratings in male to female interactions (the upper right panel), and female to male interactions (the lower left panel) - in this (mismatching) FM case, it even obtains the highest close friend rating. Again, this suggests that the GTA hyung may, depending on the situation, have an extended semantic effect of signaling close, non-romantic relationships in general (somewhat irrespective of speaker and hearer gender).

We have so far examined results on perceived relationships between the speaker and the hearer. Let us now turn to the results on perceived speaker characteristics, as elicited by the GTAs. In the interest of space, we focus on ratings for just two of the traits investigated, namely, the tultul rating and the aegyo rating. Figure 6 summarizes the key results, with the left side representing the tultul rating, and the right side, the aegyo rating.

Figure 6 captures complex interactions between GTA type and interlocutor genders (match type). First, as shown in the tutul ratings (the left side), the potential for hyung (the prescriptively male to male addressing term; pink bars) to signal tultul-ness (a kind of chill, laid back, and non-prissy attitude/trait) appears to be amplified specifically in the case of female to male interactions (the bottom left panel). That is to say, while hyung in general tends to signal tultul-ness, it is much more likely to do so (i.e., this tendency is boosted further) when female speakers choose to use the speaker mismatching hyung to address males.

Second, as shown in the aegyo ratings (the right side), the potential for unnie (the prescriptively female to female addressing term; yellow bars) and oppa (the prescriptively female to male addressing term; blue bars) to signal aegyo (a kind of infantile, feminized cuteness), appears to be neutralized in the case of male to female interactions (the top right panel). That is to say, while unnie and oppa (both of which prescriptively index female speakers) in general tend to signal aegyo, it is much less likely to do so when male speakers choose to use the speaker mismatching or speaker/hearer mismatching unnie or oppa to address females. 


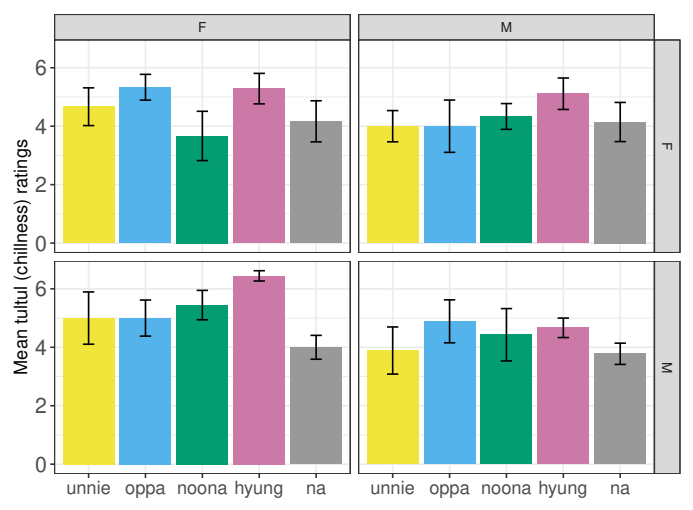

(a) Tultul ratings

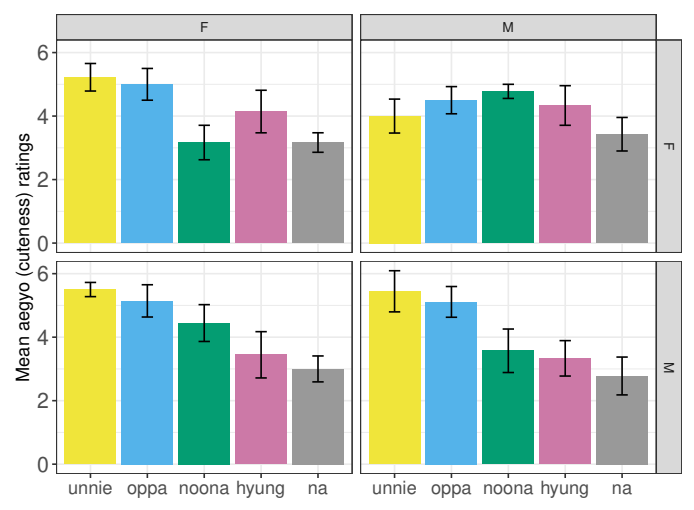

(b) Aegyo ratings

Figure 6. Likely characteristics of the speaker Legend: unnie: oppa: noona: hyung: ssi:

In sum, there appears to be significant interactions between GTA type and interlocutor gender (and/or gender/GTA mismatch types) in signaling certain character traits.

5.2.4. FREE RESPONSES \& ADDITIONAL COMMENTS. Before closing off the discussion of the experiment, we briefly examine participants' free response comments to (15) and (16), which help clarify possible motivations for choosing or avoiding a particular GTA. First, a few sample answers to matching GTA use scenarios are presented in (17)-(19). Sample answers to ssi uses are also provided in (20).

(17) unnie (FF): "In almost all situations when a younger female addresses an older female"; "When a female speaker wants to express closeness to an older female hearer"

(18) oppa (FM): "I use it to call a close male superior"

(19) noona (MF): "I use it when we are somewhat close"

(20) ssi (NA): "when talking about official matters"; "when I want to keep some distance"; "at a blind date"

These comments all confirm that the 4 GTAs are frequently used in prescription matching ways to address someone close.

Turning to the more interesting cases of mismatching uses of GTAs, a few noteworthy comments left by the participants regarding these scenarios are provided in (21)-(22), broken down into GTA type and mismatch type.

unnie (FF):

a. $\quad$ Speaker mismatch (MF): "when I (M) was the only male in the group, I found myself calling the group members unnie"; "when a shop assistant accosts a customer"

b. Hearer mismatch (FM): "I use it to call a very feminine, close male friend of mine"

c. Both mismatch (MM): "I heard a male beauty youtuber use it (addressing possibly male audience)"; "I've heard it often in LGBTQ youtube channels"

(22) hyung (MM): 
a. $\quad$ Speaker mismatch (FM): "people use hyung because oppa is cringe-y"; "I heard that up until the class of 2010-ish in campus, people used it a lot in these situations, but I haven't heard it recently myself"'

b. Hearer mismatch (MF): "to call a close female friend"; "I heard a close friend of mine use it to address a female friend of his"

Regarding unnie (the prescriptively female to female addressing term), a few participants remark that they have heard of it being used by males in certain gay communities, or when male clerks are addressing female customers in clothing or cosmetics stores. Regarding hyung (the prescriptively male to male addressing term), a few participants remark that female speakers seem to opt for hyung to address males because the prescriptively appropriate oppa has come to be inevitably associated with romantic or cringe-y connotations. Some also suggest that female social activists of a certain generation frequently used the term hyung to address both males and females, while noting that this is no longer as common in younger communities. More generally, terms prescriptively indexing same-gender relations (female to female unnie, male to male hyung) appear to be used in mismatching ways to signal solidarity or seek an in-group status in a female dominant (e.g., beauty industry) or male dominant community.

Among the observations summarized above, the potentially cringe-inducing connotations of oppa has been frequently brought up in media as well. For instance, in an interview, a female K-pop star Sejeong remarked that she calls her male colleagues hyung, because she finds oppa toe curling. In a similar vein, a female interviewer Jaejae chooses to call a male interviewee in her show as O-P-P-A (spelling out each letter in English, likely to avoid saying oppa).

6. Discussion. Let us take stock and seek to address the questions we started out with in sec. 4. The experimental results confirm that all 4 GTAs signal close relationships between the speaker and the hearer and thus differ from alternative terms of addresses like ssi. At the same time, they also demonstrate that the 4 GTAs are each associated with unique indexical fields consisting of complex relational and characterological meanings. Certain meanings are more intrinsically linked to a particular GTA (like the association between romantic relationship and oppa), whereas others may be selectively activated depending on the use of a particular GTA in particular gender pairings (e.g., the association between agyo and unnie/oppa only in non male-to-female relations). Finally, the results also suggest that mismatching GTAs may be used either to avoid the indexical baggage of matching GTAs (e.g., romantic and feminine connotations of oppa), and/or to access the positive extended meanings of mismatching GTAs (e.g., the use of hyung by female speakers to convey a chill, tough (i.e., tultul) attitude, or the use of unnie by male speakers to convey feminine solidarity).

The indexical fields associated with the GTAs, be they used in matching or mismatching ways, appear to have ideological underpinnings. For instance, it is likely no coincidence that oppa tends to signal romantic relationships and hyung, close non-romantic friendships. Although in reality, various relationships may exist between genders of all kinds, ones that are valued, emphasized, and saliently portrayed in the past and current Korean society tend to be skewed and constrained by gender ideologies (this state of affairs is somewhat evocative of the discussion surrounding the Bechtel test): heterosexual romantic relationships are emphasized for females, and masculine, platonic friendships ("bromance") for males. Likewise, certain speaker traits indexed by the GTAs, e.g., aegyo (cuteness) and oppa, and tultul (chillness) and hyung, appear to have non-arbitrary relationships with traditional gender roles. In this sense, 
gender ideologies appear to constrain or at least influence the envelope of the emerging indexical fields associated with the GTAs. Seen under this light, mismatching uses of GTAs can be construed as speakers' strategy to break away from these gender norms underlying the matching uses of GTAs, such as the sexualization of female-male relations and cute femininity (in the case of oppa), or their strategy to actively embody non-traditional gender roles connoted by mismatching uses of GTAs, such as feminine solidarity (in the case of unnie used by males).

At the same time, we also think that there exists some room for introducing and negotiating new meaning potentials of the GTAs, the key battleground being specific utterances grounded in interactions (Kiesling 2004, 2009; Podesva 2011; i.a.). To give just one example, we notice a recent trend in which female speakers use the term unnie to signal positive, empowered traits, and relationships predicated on solidarity (in the past, we believe these intentions would have likely triggered the use of the mismatching GTA hyung by the female speakers). We think that this not only reflects shifting ideas about female roles and female to female relationships, but also indicates that the accumulation of unnie uses in specific, positive contexts may have actively helped the term acquire new dimensions of meaning, and in turn maybe even helped shift ideas about female roles and female to female relationships.

This work was limited in the number and the diversity of participants recruited for the experiment. In the future, we aim to run follow-up studies focusing more on LGBTQIA communities, because the comments elicited from the present study suggest that they are at the forefront of initiating innovative, mismatching uses of the GTAs documented here. Of particular interest may be people who self-identify as non-binary; we are curious to find how they would navigate a language system which prescriptively enforces binary gender.

7. Supplementary materials. Links to experiments, data, and model summaries can be found in the following repository: https://github.com/sunwooj/genderedTAs.

\section{References}

Campbell-Kibler, Kathryn. 2007. Accent, (ing), and the social logic of listener perceptions. American speech 82(1). 32-64. https://doi.org/10.1215/00031283-2007-002.

Eckert, Penelope. 2008. Variation and the indexical field. Journal of Sociolinguistics 12(4). 453-476. https://doi.org/10.1111/j.1467-9841.2008.00374.x.

Eckert, Penelope \& Sally McConnell-Ginet. 2013. Language and gender. Cambridge University Press.

Kiesling, Scott F. 2004. Dude. American speech 79(3). 281-305. https://doi.org/10.1215/00031283-79-3-281.

Kiesling, Scott Fabius. 2009. Style as stance. In Stance: sociolinguistic perspectives, 171-194. Oxford, UK: Oxford University Press. https://doi.org/10.1093/acprof:oso/9780195331646.003.0008.

Kim, Minju. 2008. On the semantic derogation of terms for women in Korean, with parallel developments in Chinese and Japanese. Korean Studies 32(1). 148-176. https://doi.org/10.1353/ks.0.0000.

Moon, Kyuwon. 2017. Phrase final position as a site of social meaning: phonetic variation among young Seoul women: Stanford University PhD dissertation.

Podesva, Robert. 2011. Salience and the social meaning of declarative contours: Three case studies of gay professionals. Journal of English Linguistics 39(3). 233-264. https://.doi.org/10.1177/0075424211405161. 